Pacific Journal of Mathematics

COMPACT SOBOLEV IMBEDDING FOR UNBOUNDED 


\title{
COMPACT SOBOLEV IMBEDDINGS FOR UNBOUNDED DOMAINS
}

\author{
ROBERT A. ADAMS
}

\begin{abstract}
A condition on an open set $G \subset E_{n}$ which is both necessary and sufficient for the compactness of the (Sobolev) imbedding $H_{0}{ }^{u+1}(G) \rightarrow H_{0}^{m}(G)$ is not yet known. C. Clark has given a necessary condition (quasiboundedness) and a much stronger sufficient condition. We show here that (unless $n=1$ ) quasiboundedness is not sufficient, and answer in the negative a question raised by Clark on whether the imbedding can be compact if $\partial G$ consists of isolated points. We also substantially weaken Clark's sufficient condition so as to include a wide class of domains with null exterior. The gap between necessary and sufficient conditions is thus considerably narrowed.
\end{abstract}

Let $G$ be an open set in Euclidean $n$-space, $E_{n}$. Let $H_{0}^{m}(G)$ for each nonnegative integer $m$ denote the Sobolev space obtained by completing with respect to the norm

$$
\|u\|_{m, G}=\left\{\sum_{|\alpha| \leqq m} \int_{G}\left|D^{\alpha} u(x)\right|^{2} d x\right\}^{1 / 2}
$$

the space $C_{0}^{\infty}(G)$ of all infinitely differentiable complex valued functions having compact support in $G$. Here, as usual, $\alpha=\left(\alpha_{1}, \cdots, \alpha_{n}\right)$ is an $n$-tuple of nonnegative integers; $|\alpha|=\alpha_{1}+\cdots+\alpha_{n}$, and $D^{\alpha}=D_{1}^{\alpha_{1}} \cdots$ $D_{n}^{\alpha_{n}}$ where $D_{j}=\partial / \partial x_{j}, j=1, \cdots, n$.

We shall say that $G$ has the Rellich property if for each integer $m \geqslant 0$ the imbedding mapping $H_{0}^{m+1}(G) \rightarrow H_{0}^{m}(G)$ is compact. It is well known that any bounded $G$ has this property. An unbounded domain $G$ is called quasibounded if dist $(x, \partial G) \rightarrow 0$ whenever $x$ tends to infinity in $G$. If $G$ is unbounded and not quasibounded then it contains an infinite number of mutually disjoint, congruent balls. If $\varphi$ is infinitely differentiable, has support in one of these balls, and has nonzero $L^{2}(G)$ norm then the set of its translates with supports in the other balls provides a counterexample showing the imbedding $H_{0}^{1}(G) \rightarrow H_{0}^{0}(G) \equiv L^{2}(G)$ is not compact. Thus for an unbounded domain quasiboundedness is necessary for the Rellich property.

In [2] Clark showed that the following Condition 1 is sufficient to guarantee that $G$ has the Rellich property.

Condition 1 . To each $R \geqq 0$ there correspond positive numbers $d(R)$ and $\delta(R)$ satisfying

(a) $d(R)+\delta(R) \rightarrow 0$ as $R \rightarrow \infty$, 
(b) $d(R) / \delta(R) \leqq M<\infty$ for all $R$,

(c) for each $x \in G$ with $|x|>R$ there exists $y$ such that $|x-y|<d(R)$ and $G \cap\{z:|z-y|<\delta(R)\}=\varnothing$.

This condition is considerably stronger than quasiboundedness. It implies, for example, that $G$ has nonnull exterior. In [3] Clark gave an example of an unbounded domain having the Rellich property but not satisfying Condition 1. His example was the "spiny urchin," an open connected set in $E_{2}$ obtained by removing from the plane all points whose polar coordinates $(r, \theta)$ satisfy for any $k=1,2, \cdots$ the two restrictions $r \geqq k$ and $\theta=2^{-k} m \pi, m=1,2, \cdots, 2^{k+1}$.

In this paper the gap between quasiboundedness as a necessary condition and Condition 1 as a sufficient condition for a domain to have the Rellich property is narrowed from both ends. On the one hand we show that if $n \geqq 2$ then no open set whose boundary consists only of isolated points with no finite accumulation point can have the Rellich property. This settles a question raised by Clark in [3]. On the other hand we show that Condition 1 can be replaced by the following weaker Condition 2, which is still sufficient to guarantee that $G$ has the Rellich property. In the statement $B_{r}(x)$ denotes the open ball of radius $r$ about $x$.

Condition 2. There exists $R_{0} \geqq 0$ such that to each $R \geqq R_{0}$ there correspond numbers $d(R), \delta(R)>0$ such that

(a) $d(R)+\delta(R) \rightarrow 0$ as $R \rightarrow \infty$,

(b) $d(R) / \delta(R)<M \leqq \infty$ for all $R \geqq R_{0}$,

(c) for each $x \in G$ such that $|x|>R \geqq R_{0}$ the ball $B_{3 d(R)}(x)$ is disconnected into two open components $C_{1}$ and $C_{2}$ by an $n-1$ dimensional manifold forming part of the boundary of $G$ in such a way that each of the two open sets $C_{i} \cap B_{d(R)}(x), i=1,2$, contains a ball of radius $\delta(R)$.

Roughly speaking if the $n-1$ dimensional manifolds in the boundary of $G$ are reasonably smooth and unbroken, and bound a quasibounded domain (containing $G$ ) then $G$ will satisfy Condition 2. Clark's "spiny urchin" is an example of such a domain. If $n=1$ any quasibounded domain satisfies Condition 2, (but not necessarily Condition 1) and so in this case quasiboundedness is necessary and sufficient for the Rellich property.

Our principal results are as follows

THeOREM 1. If $G$ is open in $E_{n}, n \geqq 2$, and the boundary of $G$ consists only of isolated points with no finite accumulation point, then the imbedding $H_{0}^{1}(G) \rightarrow L^{2}(G)$ is not compact. Thus quasibounded- 
ness is not sufficient to guarantee the Rellich property.

THEOREM 2. If $G$ is open in $E_{n}$ and satisfies Condition 2 then it has the Rellich property.

For the proof of Theorem 1 we require the following

Lemma 1. Given $\rho, \delta>0, x_{0} \in E_{n}(n \geqq 2)$, there exists a function $u \in C^{\infty}\left(E_{n}\right)$ with the following properties

(1) $u(x)=0$ in a neighbourhood of $x_{0}$

(2) $0 \leqq u(x) \leqq 1$ for all $x$

(3) $u(x)=1$ outside the ball $B_{\rho}\left(x_{0}\right)$

(4) $\int_{E n}|\nabla u(x)|^{2} d x^{2} \leqq \delta^{2}$.

Proof. Let $f \in C^{\infty}(R)$ satisfy $0 \leqq f(t) \leqq 1, f(t)=1$ for $t \geqq 1$ and $f(t)=0$ in a neighbourhood of $t=0$. Let $m$ be a positive integer, put $r=\left|x-x_{0}\right|$ and define

$$
u(x)=v(r)=f\left([r / \rho]^{1 / m}\right) .
$$

Clearly $u \in C^{\infty}\left(E_{n}\right)$ and satisfies (1), (2) and (3). Also

$$
|\nabla u(x)|^{2}=\sum_{i=1}^{n}\left|D_{i} u(x)\right|^{2}=\left|v^{\prime}(r)\right|^{2} .
$$

Denoting by $\omega_{n}$ the surface area of the unit sphere in $E_{n}$ and making the change of variables $t=(r / \rho)^{1 / m}$ we obtain

$$
\begin{aligned}
\int_{E n}|\nabla u(x)|^{2} d x & =\omega_{n} \int_{0}^{0}\left|\frac{d}{d r} f\left(\left[\frac{r}{\rho}\right]^{1 / m}\right)\right|^{2} r^{n-1} d r \\
& =\omega_{n} \rho^{n-2} m^{-1} \int_{0}^{1}\left|\frac{d}{d t} f(t)\right|^{2} t^{1+m(n-2)} d t \\
& \leqq \omega_{n} \rho^{n-2} m^{-1}[2+m(n-2)]^{-1} \sup _{0 \leqq t \leqq 1}\left|f^{\prime}(t)\right|^{2}
\end{aligned}
$$

which, for $n \geqq 2$, can be made less than $\delta^{2}$ for a suitably large choice of $m$.

REMARK. If $\varphi \in C_{0}^{\infty}\left(E_{n}\right)$ and $u$ is constructed as above, then $\varphi \cdot u \in C_{0}^{\infty}\left(E_{n}-\left\{x_{0}\right\}\right) \subset H_{0}^{1}\left(E_{n}-\left\{x_{0}\right\}\right)$.

Proof of Theorem 1. Let $Q$ be a fixed open ball in $E_{n}$. Let $\varphi \in C_{0}^{\infty}(Q)$ be extended to all of $E_{n}$ so that $\varphi(x)=0$ in $E_{n}-Q$. Suppose $\varphi(x) \geqq 0$ for all $x$ and

$$
\|\varphi\|_{0, E_{n}}=C>0, \quad\|\varphi\|_{1, E_{n}}=K>0 .
$$


There exists $M>0$ such that for all $x$ in $E_{n}$

$$
|\varphi(x)| \leqq M, \quad\left|D_{j} \varphi(x)\right| \leqq M, \quad j=1, \cdots, n .
$$

If $Q$ contains no boundary points of $G$ put $\psi=\varphi$. Otherwise $Q$ contains only a finite number of boundary points of $G$, say $x_{1}, \cdots, x_{k}$. For $i=1, \cdots, k$ let $B_{i}=B_{\rho_{i}}\left(x_{i}\right)$ where $\rho_{i}$ is small enough that vol. $B_{i} \leqq(C / 2 k M)^{2}$. Let $\delta=K / M k$ and let $u_{i}$ be the function constructed as in Lemma 1 corresponding to the point $x_{i}$ and the constants $\rho_{i}$ and $\delta$. Put $\psi=\varphi \cdot u_{1} \cdots u_{k}$. Clearly $\psi \in H_{0}^{1}\left(Q-\left\{x_{i}, \cdots, x_{k}\right\}\right) \subset H_{0}^{1}(G)$. We have

$$
\begin{aligned}
\|\psi\|_{0, G} & \geqq\|\varphi\|_{0, E_{n}}-\sum_{i=1}^{k}\|\varphi\|_{0, B_{i}} \\
& \geqq C-\sum_{i=1}^{k} M\left(\operatorname{vol} . B_{i}\right)^{1 / 2} \geqq \frac{1}{2} C .
\end{aligned}
$$

Also

$$
\begin{aligned}
\left\|D_{j} \psi\right\|_{0, G} & \leqq\left\|D_{j} \varphi\right\|_{0, E_{n}}+\sum_{i=1}^{k}\left\|\varphi u_{1} \cdots D_{j} u_{i} \cdots u_{k}\right\|_{0, B_{i}} \\
& \leqq K+k M \delta=2 K
\end{aligned}
$$

Since $\|\psi\|_{0, G} \leqq\|\varphi\|_{0, G}=C$ we have

$$
\|\psi\|_{1, G} \leqq\left(C^{2}+4 n K^{2}\right)^{1 / 2}=C_{1} .
$$

Now let $\left\{Q_{i}\right\}_{i=1}^{\infty}$ be a family of mutually disjoint open balls in $E^{n}$ all congruent to $Q$. Let $\varphi_{i}$ be a translate of $\varphi$ with support in $Q_{i}$ and let $\psi_{i} \in H_{0}^{1}(G)$ be constructed from $\varphi_{i}$ as above, so that

$$
\left\|\psi_{i}\right\|_{0, G} \geqq \frac{C}{2}, \quad\left\|\psi_{i}\right\|_{1, G} \leqq C_{1} .
$$

Then the sequence $\left\{\psi_{i}\right\}_{i=1}^{\infty}$ is bounded in $H_{0}^{1}(G)$ but contains no subsequence convergent in $L^{2}(G)$ since for $i \neq j\left\|\psi_{i}-\psi_{j}\right\|_{0, G} \geqq C / \sqrt{2}$. Thus the imbedding $H_{0}^{1}(G) \rightarrow L^{2}(G)$ is not compact.

The proof of Theorem 2 is based on the following generalization of Poincaré's inequality which is a variant on those forms appearing in Agmon [1] and Clark [2].

Lemma 2. Let $G$ be open in $E_{n}$ and satisfy Condition 2. Let $G_{R}$ denote $G \cap\{x:|x|>R\}$. Then there exists a constant $c$ depending only on $n$ and $M$ (the constant of Condition 2 (b)) such that for all. $R \geqq R_{0}$ and every $u \in H_{0}^{1}(G)$

$$
\int_{G_{R}}|u(x)|^{2} d x \leqq c(d(R))^{2} \int_{G}|\nabla u(x)|^{2} d x
$$


Proof. Fix $R \geqq R_{0}$ and let $d=d(R), \delta=\delta(R)$. If $\alpha=\left(\alpha_{1}, \cdots, \alpha_{n}\right)$ is an $n$-tuple of integers let $Q_{\alpha}=\left\{x \in E_{n}: \alpha_{k} n^{-1 / 2} d \leqq x_{k} \leqq\left(\alpha_{k}+1\right) n^{-1 / 2} d\right\}$. Then $E_{n}=\bigcup_{\alpha} Q_{\alpha}$. Let $\varphi \in C_{0}^{\infty}(G)$. Fix $x \in G_{R}$. Then $x \in Q_{\alpha}$ for some $\alpha$. Let $B_{d}=B_{d}(x), B_{3 d}=B_{3 d}(x)$. There exists an $n-1$ dimensional manifold forming part of $\partial G$ which disconnects $B_{3 d}$ into open components $C_{1}$ and $C_{2}$ and there exist points $y_{i} \in C_{i}(i=1,2)$ such that $B_{i}\left(y_{i}\right) \subset C_{i}$. Thus $\varphi$ can be written as $\varphi=\varphi_{1}+\varphi_{2}$ where $\varphi_{i} \in C_{0}^{\infty}(G)$ and $\varphi_{1} \equiv 0$ in $C_{2}$ while $\varphi_{2} \equiv 0$ in $C_{1}$. Since $Q_{\alpha} \subset B_{d}$ we have

$$
\int_{Q_{\alpha} \cap G_{R}}|\varphi(y)|^{2} d y \leqq \int_{C_{1} \cap B_{d}}\left|\varphi_{1}(y)\right|^{2} d y+\int_{C_{2} \cap B_{d}}\left|\varphi_{2}(y)\right|^{2} d y .
$$

If $(r, \sigma)$ and $S$ denote respectively spherical coordinates in $E_{n}$ centered at $y_{2}$ and the surface of the unit sphere about $y_{2}$ we have

$$
\begin{aligned}
\int_{C_{1} \cap B_{d}}\left|\varphi_{1}(y)\right|^{2} d y & \leqq \int_{S} d \sigma \int_{\delta}^{2 d}\left|\varphi_{1}(r, \sigma)\right|^{2} r^{n-1} d r \\
& \leqq 2 d \int_{S}\left|\varphi_{1}(t, \sigma)\right|^{2} t^{n-1} d \sigma
\end{aligned}
$$

where $t=t(\sigma)$ satisfies $\delta \leqq t \leqq 2 d$. Since $\varphi_{1}(\delta, \sigma)=0$ it follows that

$$
\begin{aligned}
\left|\varphi_{1}(t, \sigma)^{2} t^{n-1}\right| & =\left|\int_{\delta}^{t} \frac{d}{d r} \varphi_{1}(r, \sigma) d r\right|^{2} t^{n-1} \\
& \leqq(2 d)^{n} \int_{\delta}^{2 d}\left|\frac{d}{d r} \varphi_{1}(r, \sigma)\right|^{2} d r \\
& \leqq(2 d)^{n} \grave{o}^{1-n} \int_{\delta}^{2 d}\left|\frac{d}{d r} \varphi_{1}(r, \sigma)\right|^{2} r^{n-1} d r .
\end{aligned}
$$

Thus, since $d / \delta<M$,

$$
\begin{aligned}
\int_{C_{1} \cap B_{d}}\left|\varphi_{1}(y)\right|^{2} d y & \leqq(2 d)^{n+1} \delta^{1-n} \int_{S} d \sigma \int_{\delta}^{2 d}\left|\frac{d}{d r} \varphi_{1}(r, \sigma)\right|^{2} r^{n-1} d r \\
& \leqq 2^{n+1} M^{n-1} d^{2} \int_{\delta \leqq\left|y-y_{2}\right| \leqq 2 d}\left|\nabla \varphi_{1}(y)\right|^{2} d y \\
& \leqq 2^{n+1} M^{n-1} d^{2} \int_{B_{3 d}}\left|\nabla \varphi_{1}(y)\right|^{2} d y .
\end{aligned}
$$

Combining this with a similar expression for $\varphi_{2}$ we obtain

$$
\begin{aligned}
\int_{Q_{\alpha} \cap G_{R}}|\varphi(y)|^{2} d y & \leqq 2^{n+1} M^{n-1} d^{2} \int_{B_{B_{d}}}|\nabla \varphi(y)|^{2} d y \\
& \leqq 2^{n+1} M^{n-1} d^{2} \int_{Q_{\alpha}^{\prime}}|\nabla \varphi(y)|^{2} d y
\end{aligned}
$$

where $Q_{\alpha}^{\prime}$ is the union of all the sets $Q_{\alpha}$ which intersect $B_{3 d}$. There 
is a number $N$ depending only on $n$ such that any $N+1$ of the sets $Q_{\alpha}^{\prime}$ have null intersection. Summing the above inequality over all $\alpha$ for which $Q_{\alpha}$ intersects $G_{R}$ we obtain

$$
\int_{G_{R}}|\varphi(y)|^{2} d y \leqq 2^{n+1} N M^{n-1}(d(R))^{2} \int_{G}|\nabla \varphi(y)|^{2} d y .
$$

This inequality extends by completion to $H_{0}^{1}(G)$.

The remaining part of the proof of Theorem 2 is similar to Clark's proof [2, Th. 3] and is included here for completeness. First, however, let $H^{m}(G, R)$ be the completion in the norm $\|\cdot\|_{m, G \cap K_{R}}$ of the space $C_{0}^{\infty}(G, R)$ of all $C^{\infty}$ functions whose support is a compact subset of $G \cap K_{R}$ where $K_{R}=\overline{B_{R}(0)}$. Since the imbedding $H_{0}^{m+1}\left(K_{R}\right) \rightarrow H_{0}^{m}\left(K_{R}\right)$ is known to be compact [4, Chapter XIV] and since an element of $H^{m}(G, R)$ can be extended to be zero outside its support so as to belong to $H_{0}^{m}\left(K_{R}\right)$ it follows that the imbeddings $H^{m+1}(G, R) \rightarrow H^{m}(G, R)$, $m=0,1,2, \cdots$ are compact.

Proof of Theorem 2. It suffices, by an inductive argument, to prove only that the imbedding $H_{0}^{1}(G) \rightarrow L^{2}(G)$ is compact. We make use of the following well known compactness criterion for sets in $L^{2}(G)$ : if $G \subset E_{n}$ and the sequence $\left\{u_{k}\right\}_{k=1}^{\infty}$ is bounded in $L^{2}(G)$ then it is compact in $L^{2}(G)$ provided

(a) for every bounded $G^{\prime} \subset G$ the sequence $\left\{u_{k} \mid G^{\prime}\right\}$ is compact in $L^{2}\left(G^{\prime}\right)$, and

(b) for each $\varepsilon>0$ there exists $R>0$ such that for all $k$

$$
\int_{G_{R}}\left|u_{k}(x)\right|^{2} d x<\varepsilon
$$

Now let $\left\{u_{k}\right\}$ be a sequence bounded in $H_{0}^{1}(G)$, say $\left\|u_{k}\right\|_{1, G} \leqq K$. By Lemma 2 , for $R \geqq R_{0}$ we have $\left\|u_{k}\right\|_{0, G_{R}} \leqq C(d(R))^{2} K \rightarrow 0$ as $R \rightarrow \infty$ so condition (b) of the criterion is satisfied. To establish (a) let $G^{\prime}$ be a bounded subset of $G$, so that $G^{\prime} \subset K_{R}$ for some $R$. Since $\left\{u_{k} \mid K_{R}\right\}$ is bounded in $H^{1}(G, R)$ it is compact in $H^{0}(G, R)=L^{2}\left(K_{R} \cap G\right)$ and so $\left\{u_{k} \mid G^{\prime}\right\}$ is compact in $L^{2}\left(G^{\prime}\right)$. Thus $\left\{u_{k}\right\}$ is compact in $L^{2}(G)$, whence the theorem.

\section{REFERENCES}

1. Shmuel, Agmon, Lectures on elliptic boundary value problems, Van Nostrand, Princeton, 1965.

2. Colin Clark, An embedding theorem for function spaces, Pacific J. Math. 19 (1966), $243-251$.

3. - Rellich's embedding theorem for a "spiny urchin" Canad. Math. Bull. 10 (1967), 731-734. 
4. N. Dunford and J. Schwartz, Linear operators, Part II, Interscience, New York, 1963.

Received January 8, 1968. Research partially supported by National Research Council of Canada Operating Grant A-3973.

The Unversity of British COLUmbia 



\section{PACIFIC JOURNAL OF MATHEMATICS}

\section{EDITORS}

\author{
H. SAMELSON \\ Stanford University \\ Stanford, California 94305 \\ Richard Pierce \\ University of Washington \\ Seattle, Washington 98105
}

J. DUGUNDJI

Department of Mathematics

University of Southern California

Los Angeles, California 90007

BASIL GORDON*

University of California

Los Angeles, California 90024

\section{ASSOCIATE EDITORS}
E. F. BECKENBACH
B. H. NeUmanN
F. WOLF
K. YoSHIDA

\section{SUPPORTING INSTITUTIONS}

UNIVERSITY OF BRITISH COLUMBIA CALIFORNIA INSTITUTE OF TECHNOLOGY

UNIVERSITY OF CALIFORNIA MONTANA STATE UNIVERSITY

UNIVERSITY OF NEVADA

NEW MEXICO STATE UNIVERSITY

OREGON STATE UNIVERSITY

UNIVERSITY OF OREGON

OSAKA UNIVERSITY

UNIVERSITY OF SOUTHERN CALIFORNIA
STANFORD UNIVERSITY

UNIVERSITY OF TOKYO

UNIVERSITY OF UTAH

WASHINGTON STATE UNIVERSITY

UNIVERSITY OF WASHINGTON

AMERICAN MATHEMATICAL SOCIETY CHEVRON RESEARCH CORPORATION TRW SYSTEMS

NAVAL WEAPONS CENTER 


\section{Pacific Journal of Mathematics}

\section{Vol. 32, No. $1 \quad$ January, 1970}

Robert Alexander Adams, Compact Sobolev imbeddings for unbounded domains ........................................ 1

Bernhard Amberg, Groups with maximum conditions .................. 9

Tom M. (Mike) Apostol, Möbius functions of order k............... 21

Stefan Bergman, On an initial value problem in the theory of two-dimensional transonic flow patterns ................... 29

Geoffrey David Downs Creede, Concerning semi-stratifiable spaces ...... 47

Edmond Dale Dixon, Matric polynomials which are higher

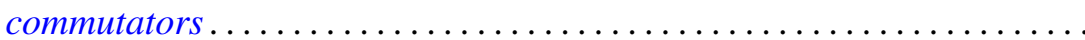

R. L. Duncan, Some continuity properties of the Schnirelmann density.

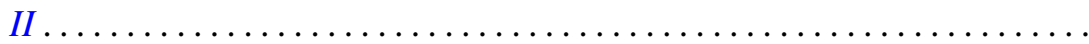

Peter Larkin Duren and Allen Lowell Shields, Coefficient multipliers of $H^{p}$

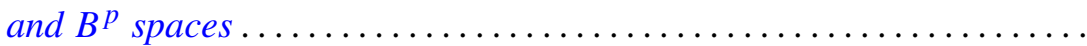

Hector O. Fattorini, On a class of differential equations for vector-valued

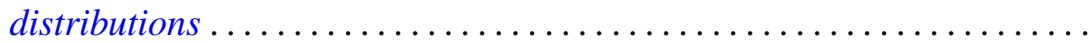

Charles Hallahan, Stability theorems for Lie algebras of derivations. . . . . . 105

Heinz Helfenstein, Local isometries of flat tori ................ 113

Gerald J. Janusz, Some remarks on Clifford's theorem and the Schur

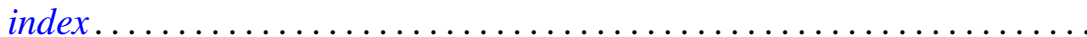

Joe W. Jenkins, Symmetry and nonsymmetry in the group algebras of discrete groups. ...

Herbert Frederick Kreimer, Jr., Outer Galois theory for separable

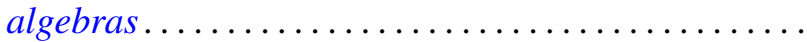

D. G. Larman and P. Mani, On visual hulls

R. Robert Laxton, On groups of linear recurrences. II. Elements of finite order.

Dong Hoon Lee, The adjoint group of Lie groups ...

James B. Lucke, Commutativity in locally compact rings

Charles Harris Scanlon, Rings of functions with certain Lipschitz

$$
\text { properties ............................... }
$$

Binyamin Schwarz, Totally positive differential systems .

James McLean Sloss, The bending of space curves into piecewise helical curves.

James D. Stafney, Analytic interpolation of certain multiplier spaces ...

Patrick Noble Stewart, Semi-simple radical classes.......

Hiroyuki Tachikawa, On left $\mathrm{QF}-3$ rings ...................... 255

Glenn Francis Webb, Product integral representation of time dependent nonlinear evolution equations in Banach spaces.... . . . 